Construire le voisin. Pratiques européennes

\title{
La dimension méditerranéenne de la politique Justice et Affaires intérieures
}

Sarah Wolff

\section{(2) OpenEdition \\ 1 Journals}

\section{Édition électronique}

URL : http://journals.openedition.org/conflits/2581

DOI : 10.4000/conflits.2581

ISSN : $1777-5345$

Éditeur :

CCLS - Centre d'études sur les conflits lilberté et sécurité, L'Harmattan

\section{Édition imprimée}

Date de publication : 17 septembre 2007

Pagination : 77-99

ISBN : 978-2-296-03458-7

ISSN : 1157-996X

\section{Référence électronique}

Sarah Wolff, "La dimension méditerranéenne de la politique Justice et Affaires intérieures », Cultures \& Conflits [En ligne], 66 | été 2007, mis en ligne le 04 janvier 2010, consulté le 30 mars 2021. URL :

http://journals.openedition.org/conflits/2581 ; DOI : https://doi.org/10.4000/conflits.2581 


\title{
La dimension méditerranéenne de la politique Justice et Affaires intérieures 1
}

\section{Sarah WOLFF}

Sarab Wolff est doctorante à la London School of Economics. Ses recherches portent sur la politique étrangère de l'UE, la dimension extérieure de la politique Justice et Affaires intérieures, le voisinage méditerranéen, avec un intérêt particulier pour l'Egypte et le Maroc. Diplômée de l'IEP de Grenoble et de l'université d'Amsterdam, elle a obtenu un Master of Science en politique européenne et gouvernance à la LSE. Assistante éditoriale du European Journal of International Relations, elle est également chargée de cours en science politique.

\section{Justice et Affaires intérieures : un objectif de politique étrangère}

\begin{abstract}
A u lendemain de la fin de la Guerre froide, les phénomènes de la « déterritorialisation » des menaces ainsi que de la reconceptualisation de la notion de frontières accompagnaient une transformation du concept de sécurité européenne ${ }^{2}$. La globalisation et la transnationalisation des menaces ont en effet provoqué une altération progressive des notions de frontières intérieures et extérieures. Les frontières contemporaines, produit de constructions sociales et de cadres cognitifs, sont le résultat de l'émergence de nouvelles communautés sécuritaires qui dressent de nouvelles frontières entre «insiders » et «outsiders " 3 . Les entités géographiques comme l'Europe définissent leurs frontières selon leur propres
\end{abstract}

1. Cet article a été initialement présenté lors de la première école doctorale du réseau Challenge "Perspectives sur la Politique européenne de voisinage », 21-22 avril 2005 au Centre for European Policy Studies (CEPS) à Bruxelles. L'auteur remercie sincèrement Federica Bicchi, William Wallace et Grégory Mounier pour leurs commentaires sur les précédentes versions de cet article, ainsi que diplomates interviewés à Bruxelles, Londres et Rabat qui lui ont fourni de précieuses informations.

2. Dans le texte original en anglais, le terme utilisé est "border », qui a une acceptation différente $\mathrm{du}$ terme de «frontier». Le premier renvoie à une perception cognitive, le second est un concept géographique.

3. "Groups of "like-minded" nations, such as the member states of the EU, are more and more conscious of an external frontier marking them off from the others ", Anderson M., Bort E., The Frontiers of the European Union, New York, Palgrave, 2001. 
perceptions et pratiques sécuritaires. Cela a conduit à l'apparition de nouveaux discours qui «ne font plus la distinction entre sécurité intérieure et sécurité extérieure », et se caractérisent par une vision large de la notion de sécurité, y incluant les questions énergétiques, les droits de l'Homme, les questions migratoires mais également le crime organisé 4 .

Cette évolution de la nature de la sécurité européenne a eu d'importantes conséquences pour la politique Justice et Affaires intérieures (JAI) de l'Union européenne (UE), qui a été développée à la fois au niveau interne et externe, afin de consolider le régime européen de sécurité intérieure. Bien que beaucoup d'études ont jusqu'ici analysé sa dimension interne ${ }^{5}$, au cœur de sa « raison d'être », peu d'attention a été portée à la dimension extérieure de cette politique initiée avec le sommet de Tampere en 1999. Reconnaissant la nécessité de recourir aux instruments de Politique étrangère et de sécurité commune (PESC) - les stratégies communes et les accords avec les pays tiers afin de renforcer la dimension extérieure de la politique JAI, entre autres -, ce sommet marque un tournant décisif dans le développement de cette politique. Par la suite, le Conseil de Feira de 2000 a précisé les orientations devant guider l'établissement de cette dimension extérieure, qui trouve en partie sa légitimité dans le principe de "parallélisme de compétences ", érigé par la jurisprudence AETR de 1971, selon laquelle les compétences internes de l'UE sont parallèles à ses compétences externes ${ }^{6}$. La Cour de justice des Communautés européennes a ainsi reconnu que "la compétence de la communauté s'étend à des relations relevant $d u$ droit international et implique, dès lors, dans le domaine visé, la nécessité d'accords avec les états tiers intéressés ». Cette logique juridique est l'un des facteurs qui explique que la politique JAI ait été intégrée de manière systématique dans les documents de politique extérieure, par exemple avec le Programme de La Haye de 2004. La stratégie pour la dimension extérieure de la politique JAI datant de 2005 a, quant à elle, consacré cette politique interne comme objectif de politique étrangère. Le document stipule ainsi que «l'UE doit [...] faire de la politique JAI une priorité centrale dans ses relations extérieures et assurer une approche coordonnée et cobérente ${ }^{7}$ ». Il ne s'agit donc plus simplement d'intégrer de manière systématique la politique JAI dans les politiques extérieures, mais plutôt d'en faire une priorité centrale de l'UE dans

4. Ibid. p. 23.

5 . Pour les études analysant la politique JAI, on peut se référer entre autres aux travaux suivants : Monar J., "The dynamics of Justice and Home Affairs: laboratories, driving factors and costs ", Journal of Common Market Studies, vol. 39, n4, p. 747, 2001 ; Den Boer M., Plural Governance and EU Internal Security: Chances and Limitations of Enhanced Cooperation in the area of Freedom, Security and Justice, ARENA, 2004 ; Lavenex S., Wallace W., " Justice and Home Affairs ", in Wallace H., Wallace W., Pollack M., Policy-Making in the EU, Oxford, Oxford Press University, 2005.

6. Arrêt de la Cour européenne de justice des communautés, cas 22/70, 31 mars 1971, Commission contre Conseil, Accord européen sur les transports routiers (AETR).

7. European Council, A Strategy for the External Dimension of JHA: Global Freedom, Security and Justice, R. JAI 417, 2005. 
ses relations avec les pays tiers. En d'autres termes, la politique JAI fait désormais partie des objectifs de la politique étrangère de l'UE.

Au regard de ces récents développements, il est pour le moins surprenant que les chercheurs en Politique étrangère européenne (PEE) aient jusqu'ici ignoré la politique JAI dans leur analyse des relations extérieures de l'UE, un domaine qui, non seulement, relève des premier et troisième piliers, mais de plus en plus du deuxième incarné par la PESC et la Politique européenne de sécurité et de défense (PESD). Smith a d'ailleurs souligné l'absence de lien entre politique JAI et PEE dans la littérature, bien que celui-ci soit de plus en plus visible ${ }^{8}$. Il est en effet frappant de constater que peu de tentatives ont été menées par les spécialistes de la PEE pour intégrer la littérature florissante portant sur la dimension extérieure de la sécurité intérieure depuis le sommet de Tampere. Pourtant, depuis cette date, la lutte contre le terrorisme et l'immigration clandestine, la gestion des frontières, et l'extension des compétences des agences en charge de la coopération policière et judiciaire telles qu'Eurojust et Europol ont été placées au cœur de l'action extérieure de l'UE 9.

La dimension extérieure de la politique JAI est devenue un objectif constant de la politique de l'UE et de ses Etats-membres. Si, à première vue, cette dimension extérieure a été analysée dans le contexte de la décentralisation de la gestion de la politique européenne d'immigration vers les pays tiers ${ }^{10}$, de récentes études

8. Smith K. E., « Understanding the European foreign policy system », Contemporary European History, vol. 12, n², 2003b, pp. 239-254, et plus particulièrement p. 244.

9. Voir Guiraudon V., « European integration and migration policy: vertical policy-making as venue shopping ", Journal of Common Market Studies, vol. 38, n², pp. 251-271, 2000 ; Stetter S., " Regulating migration: authority delegation in justice and home affairs ", Journal of European Public Policy, vol. 7, n¹, pp. 80-103, 2000; Monar J., « The dynamics of Justice and Home Affairs: laboratories, driving factors and costs", op. cit.; Thielmann E., Soft Europeanisation of Migration Policy: European Integration and Domestic Policy Change, Madison, Wisconsin, ECSA Seventh Biennial International Conference, 2001 ; Grabbe H., "Justice and Home Affairs: faster decisions, more secure rights ", New Designs for Europe, Centre for European Reform, 2002 ; Den Boer, Plural Governance and EU Internal Security: Chances and Limitations of Enhanced Cooperation in the area of Freedom, Security and Justice, Paper for ARENA, Oslo, 2004 ; Huysmans J., « A Foucaultian view on spill-over: freedom and security in the EU ", Journal of International Relations and Development, vol. 7, n³, pp. 294318, 2004 ; Monar J., "The "Area of Freedom, Security and Justice" in the EU's draft constitutional treaty and its implications for the Mediterranean ", The European Union and the Mediterranean. The Mediterranean's European Challenge, Malta, University of Malta, 2004, pp. 215-243 ; Lavenex S., Wallace W., "Justice and Home Affairs ", Policy-making in the European Union, Oxford, Oxford Press University, 2005 ; Pastore F., Friedrichs J., et al., «Is there a European strategy against terrorism? A brief assessment of supra-national and national responses », Working Paper, Roma, CeSPI, CentroStudidiPoliticaInternazionale (CeSPI), p. 26, 2005 ; Lugna L., «Institutional framework of the European Union counter-terrorism policy setting », Baltic Security and Defence Review, vol. 8, 2006, pp. 101-127 ; Zimmermann D., Terrorist Threats, the European Union and Counter-Terrorism, Zurich, Center for Security Studies, Swiss Federal Institute of Technology, 2006.

10. Voir Stetter S., "Regulating migration: authority delegation in justice and home affairs ", Journal of European Public Policy, vol. 7, ${ }^{\circ} 1$, mars 2000 ; Lavenex S., " The EU enlargement and the challenge of policy transfer: the case of refugee policy ", Journal of Ethnic and Migration Studies, vol. 28, n 4, 2002, pp. 701-721 ; Pastore F., «Just another European dream? 
attestent des liens de plus en plus forts entre politique JAI et PESC. Ces études se sont attachées à démontrer la présence d'expertise JAI au sein des missions de la PESD, et à travers des études empiriques, la coopération policière et judiciaire par-delà les frontières de l'UE, ainsi que l'étroite connivence qui existe désormais entre objectifs traditionnels de la PEE et politique JAI ${ }^{11}$. Cependant, la majorité de ces universitaires se sont concentrés sur les pays d'Europe centrale et orientale (PECO), une orientation qui est notamment le résultat du premier plan d'action JAI négocié avec l'Ukraine en 2003, ainsi que de l'intégration de l'acquis communautaire en matière de JAI par les candidats à l'élargissement. Par ailleurs, peu d'entre eux se sont attachés à conceptualiser la relation entre PEE et JAI et à analyser les conséquences que peut avoir l'intrusion de la politique JAI dans les relations extérieures de l'UE.

C'est dans ce contexte que s'inscrit cet article, dont le but est d'attirer l'attention sur les liens entre politique JAI et PESC, à travers le cas du voisinage méditerranéen. Cette région est en effet depuis 2000 l'objet de la part de l'UE d'une demande croissante de coopération en matière de terrorisme et de migrations. Arguant que la politique JAI est devenue un objectif de politique étrangère de l'UE, l'article étudie dans quelle mesure la politique JAI contribue à l'action extérieure de l'UE. En d'autres termes, pour emprunter la métaphore de Pastore, existe-t-il une réconciliation des «deux bras du Prince 12 » ou, au contraire, l'injection d'éléments JAI ne contribue-

Why did the communitarization of immigration and asylum policies fail and how we should revive it? ", European Migration and Refugee Policy: New Developments, Rome, The Cicero Foundation, 2002 ; Lavenex S., Uçarer E., « The external dimension of Europeanization. The case of immigration policies », Cooperation and Conflict, vol. 39, $\mathrm{n}^{\circ} 4,2004$, pp. 417-443; Sterkx S., « The Comprehensive Approach off Balance: Externalisation of EU Asylum and Migration Policy », Politieke Wetenschappe, ${ }^{\circ} 4$, p. 37, 2004.

11. Hurwitz A., The Externalisation of EU Policies on Migration and Asylum: Readmission Agreements and Comprehensive Approaches, 2003 ; Bigo D., "When two become one. Internal and external securitisations in Europe », in Kelstrup M., Williams M. C., International Relations Theory and the Politics of European Integration. Power, Security and Community, Londres, Routledge, 2000, pp. 171-204 ; Pastore F., « Reconciling the Prince's two "arms". Internal-external security policy coordination in the European Union », op. cit. ; Lavenex S., "EU external governance in "wider Europe" ", Journal of European Public Policy, vol. 11, n ${ }^{\circ}$, 2004, pp. 680-700 ; Lavenex S., Uçarer E., "The external dimension of europeanization. The case of immigration policies ", op. cit. ; Lutterbeck D., "Blurring the dividing line: the convergence of internal and external security in Western Europe ", European Security, vol. 14, n², 2005, pp. 231-253 ; Rees W., " The external face of internal security », in Hill C., Smith M., International Relations and the European Union, Oxford, Oxford University Press, 2005, pp. 206-224 ; Wichmann N., Promoting the Rule of Law in the ENP - Strategic or Normative Power EU?, CEPS, Brussels, 2006 ; Mounier G., « European police missions: from security sector reform to externalization of internal security beyond the borders ", HUMSEC, vol. 1, n ${ }^{\circ}$, 2007 (à venir).

12. Cité dans Pastore F., op. cit. : « Deux craintes doivent occuper un prince: l'intérieur de ses Etats et la conduite de ses sujets sont l'objet d'une; le dehors et les dessins des puissances environnantes sont celui de l'autre. Pour celle-ci, le moyen de se prémunir est d'avoir de bonnes armes et de bons amis et l'on aura toujours de bons amis quand on aura de bonnes armes: d'ailleurs, tant que le prince sera en sûreté et tranquille au dehors, il le sera aussi au-dedans, à moins qu'il n'eût été déjà troublé par quelque conjuration; et si même au-dehors quelque entreprise est formée contre lui, il trouvera dans l'intérieur [...] les moyens de résister à toute attaque, pourvu toutefois qu'il se soit conduit et qu'il ait gouverné conformément à ce que j'ai observé, et que de plus il ne perde 
t-elle pas davantage à l'incohérence de l'UE en tant qu'acteur de relations internationales ? Cet article exposera tout d'abord les approches d'externalisation et de gouvernance extérieure qui constituent une base intéressante pour conceptualiser dans le futur les liens entre JAI et PEE. Dans un second temps, une analyse empirique détaille la dimension méditerranéenne de la politique JAI et notamment l'état de la coopération policière et judiciaire (CPJ) avec les voisins méditerranéens ; avant de montrer que ce mode particulier de gouvernance a été institutionnalisé au sein de la PEV, comme l'illustre le cas du Maroc. La dernière partie met en perspective cette analyse empirique de l'intégration des questions JAI dans l'action extérieure de l'UE à travers une discussion sur les conséquences que de tels développements ont sur la cohérence de l'action extérieure de l'UE.

\section{Externalisation et gouvernance extérieure}

Comme nous l'avons vu, le développement d'une dimension extérieure de la politique JAI n'est pas sans poser quelques défis à la recherche portant sur l'intégration européenne. Comment expliquer le fait qu'une politique initialement conçue autour d'objectifs internes poursuive désormais des objectifs externes? Intuitivement, les universitaires qui se sont penchés sur la question se sont tournés vers les théories néo-fonctionnalistes et institutionnalistes pour expliquer ce phénomène dit d' « externalisation de la politique JAI 13 ».

Le terme d'externalisation reflète deux réalités : l'externalisation vers les pays tiers, mais également l'externalisation d'une politique interne vers la politique extérieure de l'UE. L'argument avancé selon lequel l'UE « exporte » ou « externalise » un modèle endogène tel que sa politique de sécurité intérieure, a été développé par Sandra Lavenex. L'UE ayant la volonté d'étendre «ses frontières légales » tout en n'offrant à ses voisins qu'un accès limité aux «frontières institutionnelles » de l'UE, Lavenex explique qu'elle «extraterritorialise » ses politiques internes vers les pays tiers ${ }^{14}$. Le concept d'externalisation comme processus néofonctionnaliste a été avancé dès 1969 par Philippe Schmitter ${ }^{15}$. Considérant les conditions extérieures comme données, l'hypothèse assume que les unités régionales telles que l'UE «se trouveront de plus en plus contraintes - quelques soient les intentions premières - d'adopter des politiques communes vis-à-vis des parties tiers non prenantes $16 »$. Selon la logique de l'effet «spillover», la collaboration

point courage ", Machiavel N., Le Prince, chap. XIX «Qu'il faut éviter d'être méprisé et haï », 1532, trad. française par J.V. Périès, 1825.

13. Par mesure de clarté, bien qu'une terminologie différente soit utilisée par la Commission européenne (l'ancienne DG JAI s'intitule désormais DG Justice, Liberté et Sécurité), cet article n'utilise que l'expression traditionnelle de «Justice et Affaires intérieures».

14. Lavenex S., "EU external governance in "wider Europe" », op. cit., p. 684 ; Lavenex S., Uçarer E., op. cit.

15. Schmitter P., "Three neo-functional hypotheses about international integration", International Organization, vol. 23, $\mathrm{n}^{\circ} 1,1969$, pp. 161-166.

16. Schmitter P., Neo-Neo Functionalism: Déjà Vu, all over again?, Florence, European University Institute, 2002, p. 16. 
des Etats-membres autour de politiques communes les amène naturellement à coopérer sur les dimensions extérieures de ces politiques, ce qui conduit donc à une «externalisation» des politiques internes. Cette «hypothèse de l'externalisation » affirme que "les conditions externes seront de moins en moins déterminées de manière exogène si des stratégies intégratices plutôt que désintégratices sont adoptées en commun 17 ». En d'autres termes, plus l'intégration entre Etatsmembres est intense, moins l'environnement extérieur aura d'influence.

En outre, les pressions extérieures seront absorbées

« jusqu'à ce que des négociations conjointes vis-à-vis des outsiders forment partie intégrale du processus de décision, au point que le système international accorde à la nouvelle unité, le statut de participant à part entière 18 ».

Le processus d'externalisation des politiques internes doit donc naturellement conduire à l'intégration des outsiders dans l'unité régionale. L'UE externalise ses politiques à ses voisins car "l'intégration produit des effets négatifs sur les acteurs hors de l'UE, et que leur candidature d'adhésion doit être comprise comme une réponse directe à cette peur d'exclusion de la coopération européenne 19 ». Cette discrimination envers les outsiders fournit à l'UE le «stimulus externe » nécessaire pour ouvrir ces politiques à la participation de ses voisins. Schmitter prédit en effet que les outsiders insisteront afin d'assumer plus de responsabilité, notamment dans les domaines de la défense et de la sécurité 20 .

Cependant, un des défauts de l'approche néo-fonctionnaliste est de considérer la relation entre liberté et sécurité comme fonctionnelle et résultant d'une compétition politique. Le problème de cette interprétation est le suivant :

« elle n'explique pas le résultat de cette compétition politique au sujet de ce qui constitue un équilibre acceptable entre liberté de mouvement et maintien de l'ordre au sein du marché commun ; elle n'explique pas réellement l'étendue et la portée du domaine de la sécurité intérieure 21 ».

Alternativement, l'export de modèles endogènes en direction des pays tiers a été étudié par l'approche dite «de gouvernance extérieure de l'UE». Traditionnellement, le concept de « gouvernance» se retrouve dans l'analyse des

17. Ibid., p. 16.

18. Ibid.

19. Sjursen H., «Enlargement and the common foreign and security policy: transforming the EU's external policy? », Working Paper 18, ARENA, 1998.

20. Schmitter P., op. cit., pp. 23-24.

21. Huysmans J., "A Foucaultian view on spill-over: freedom and security in the EU », op. cit., p. 297. 
politiques internes de l'UE et a permis d'identifier de nouveaux « modes de gouvernance » tels que la méthode ouverte de coordination, par exemple ${ }^{22}$. A ce stade de la recherche, il est aisé de distinguer deux branches de la littérature sur la gouvernance extérieure de l'UE ${ }^{23}$. La première porte sur l'export des systèmes de gouvernance de l'UE envers les pays tiers, c'est-à-dire sur le contenu de ce qui est exporté, tel que l'acquis communautaire ${ }^{24}$. La seconde branche se concentre sur la forme que ce transfert de règles revêt. Suivant cette dernière approche, Sedelmeier et Schimmelfennig ont identifié les principaux traits de la transmission de règles 25 envers les pays d'Europe centrale et orientale (PECO) et évalué quel mode de gouvernance se révélait le plus efficace ${ }^{26}$. Dans le contexte de l'Europe élargie, la gouvernance extérieure permet à l'UE de "s'attaquer aux interdépendances à travers la projection externe de solutions internes 27 » et d'inclure les pays tiers dans la poursuite d'objectifs internes à l'UE. En ce sens, en exerçant sa gouvernance extérieure, l'UE poursuit deux types d'objectifs : les premiers relèvent de la politique étrangère, et les seconds de la politique interne. Ainsi, le transfert de politiques dans le domaine des politiques commerciales et de la JAI aide à " accrôtre l'efficacité et à résoudre des problèmes de capacité des politiques internes de l'UE», tandis que le transfert de politiques dans le domaine de la démocratisation et des droits de l'Homme sert des objectifs de politique étrangère tels que «shaping the milieu 28 ".

Proposant un agenda de recherche sur la gouvernance extérieure de l'UE, Schimmelfennig et Wagner soutiennent que cette approche comprend une certaine valeur ajoutée si les étapes suivantes sont démontrées : premièrement, dans quelle mesure la politique extérieure de l'UE est influencée par la gouvernance multiniveaux, et deuxièmement, dans quelle mesure ce processus véhicule des «effets transformateurs » pour les institutions européennes et celles des Etats-membres 29. Ces effets transformateurs peuvent êtres externes (par exemple, comment la gou-

22 . L'approche dite de la " gouvernance » a été appliquée au processus décisionnel de l'UE, notamment à travers l'analyse de la gouvernance multi-niveaux (voir Hooghe L., Marks G., « Multilevel governance and European integration ", Lanham, Rowman and Littlefield) et la présence de réseaux de gouvernance (voir Kohler-Koch B., Linking EU and National Governance, Oxford, Oxford Press University, 2003).

23. Schimmelfennig F., Sedelmeier U., « Governance by conditionality: EU rule transfer to the candidate countries of Central and Eastern Europe ", Journal of European Public Policy, vol. 11, $n^{\circ} 4,2004$, pp.661-679.

24. Kohler-Koch B., "Catching up with change. The transformation of governance in the European Union », op. cit., vol. 3, n³, 1996, pp. 359-380.

25. Dans le texte original «rule transfer».

26. Schimmelfennig F., Sedelmeier U., op. cit., p. 670.

27 . Lavenex S., "EU external governance in "wider Europe" ", op. cit., p. 695.

28 . «Shaping the milieu» est un objectif de politique étrangère qui consiste à «changer le système international, et inclut des choses comme le renforcement du droit international et des organisations multilatérales ». En poursuivant de tels objectifs, les Etats-nations «créent un environnement favorable à leurs intérêts », voir Hyde-Price A., «The EU, power and coercion: from "civilian" to "civilising" power ", From Civilian to Military Power: the European Union at Crossroads?, Oslo, CIDEL Workshop, 2004.

29. Schimmelfennig F., Wagner W., «External governance in the European union », Journal of European Public Policy, vol. 11, n 4, 2004, pp. 657-660, voir p. 658. 
vernance extérieure de l'UE influence les pays tiers), mais également interne, sur la transversalité des piliers communautaires par exemple («cross-pillarization ») 30 .

Le phénomène d'externalisation, compris comme l'expansion de l'acquis communautaire aux pays tiers, a été analysé dans les cas de la lutte contre le terrorisme ${ }^{31}$, de la politique JAI, de l'énergie et de l'environnement ${ }^{32}$. Bien que déjà appliqué au cas des $\mathrm{PECO}$, le défi est désormais d'analyser ce phénomène à une autre échelle, celle de l'expansion des modèles et politiques européens aux pays voisins qui n'ont pas la perspective de devenir un jour membres de l'UE. Le but de cet article est de mettre en lumière le contenu et la forme de la gouvernance extérieure de l'UE dans le domaine JAI, mais également d'en tirer des conséquences pour l'UE en tant qu'acteur de relations internationales.

\section{Les aspects multilatéraux de la coopération policière et judicaire en médi- terranée}

Au niveau européen, la Coopération policière et judiciaire (CPJ) symbolise la coopération transgouvernementale ${ }^{33}$ caractérisée par une mise en réseaux intense, la mise en commun d'informations et l'échange de meilleures pratiques. Les agences coercitives ${ }^{34}$ en sont les acteurs principaux, leur coordination tombant désormais sous la houlette d'agences européennes semi-autonomes et d'institutions telles qu'Europol, Eurojust, l'Ecole européenne de la police (CEPOL), ou bien encore la toute récente Agence européenne pour la gestion de la coopération opérationnelle aux frontières extérieures (FRONTEX). La coopération transgouvernementale demeure toutefois importante. Elle est à l'origine de nombreux accords bilatéraux et multilatéraux, ainsi que de conventions comme la convention de Prüm, signée récemment entre sept Etats-membres afin de faciliter la coopération policière transfrontalière, et qui fait déjà figure de Schengen III 35. L'information est réunie dans plusieurs bases de données : EURODAC permet de comparer les empreintes des demandeurs d'asiles et des immigrants illégaux ; le Système d'information de Schengen (SIS), un réseau d'in-

30. Stetter S., «Cross-pillar politics: functional unity and institutional fragmentation of EU foreign policies ", Journal of European Public Policy, vol. 11, n4, 2004, pp. 720-739, voir p. 721

31. Longo F., The Externalisation of the Fight against Transnational Organisd Crime: a New Aspect of the EU's International Actorness, ECPR Joint Sessions, Workshop on Understanding the EU's international presence Grenoble, 2001.

32. Lavenex S., " EU external governance in “wider Europe” ", op. cit.

33. Helen Wallace parle de "transgouvernmentalisme intensif " ( intensive transgovernmentalism »), selon lequel les gouvernements des Etats-membres se sont engagés dans l'intégration européenne, mais qui considèrent que dans certain cas que «le cadre institutionnel de l'UE [est] inapproprié ou inacceptable, ou bien pas encore mûr pour être adopté ", voir Wallace H., Wallace W., et al., Policy-Making in the European Union, op. cit.

34. Le terme d' "agences coercitives" proposé par Challenge est ici utilisé comme équivalent de «law enforcement agencies» qui recouvre les agences responsables de l'application de la loi/de la répression, telles que les agences judiciaires et policières.

35. Balzacq T., Bigo D., et al., "Security and the Two Level Game: the Treaty of Prüm, the EU and the Management of Threats », Working Document, CEPS. Brussels, 2006, p. 28. 
formation permettant aux autorités compétentes d'accéder à des bases de données sur des individus, des véhicules ou bien des objets perdus ou volés; et enfin le VIS, le Système d'information sur les visas. Cet échange d'information a lieu dans différents domaines (trafic de drogue, CPJ en matière civile et criminelle, immigration) et constitue un exemple de cross-pillarisation, qui évolue entre intergouvernementalisme et politiques communautaires et, par conséquent, tombe sous le coup de divers modes de décision.

Bien que ne faisant pas l'objet de cet article, il est tout de même nécessaire de souligner que les préoccupations JAI vis-à-vis des partenaires méditerranéens sont d'abord apparues autour des questions de migration. Originellement confinés durant les années 1960 et 1970 aux anciens pouvoirs coloniaux européens, la nouvelle donne de l'après Guerre froide a changé fondamentalement les flux migratoires. L'expression de «Rio Grande de l'UE 36 » souligne le défi considérable que représentent les questions démographiques et migratoires pour l'UE. Bien que les migrations méditerranéennes soient un phénomène historique, celles-ci sont devenues bien plus complexes, dessinant de nouvelles lignes de fracture entre insiders et outsiders. L'inclusion de Chypre et Malte, deux anciens partenaires méditerranéens, les négociations avec la Turquie, ainsi que le nouvel environnement géopolitique qui a suivi la chute du communisme et les guerres dans les Balkans occidentaux ont transformé en profondeur le profil et les routes des migrants. L'Espagne, le Portugal, l'Italie, la Grèce mais aussi Malte et Chypre sont devenus les nouvelles destinations des embarcations de fortune en provenance d'Afrique du Nord et du Moyen-Orient. En 2003, les interceptions se sont élevées à 14000 en Italie, 11000 en Espagne et environ 4000 en Grèce en 2002 37. Quant à Malte, les garde-côtes ont intercepté 1700 immigrants illégaux en 2003 et, en 2006, 100 immigrants étaient détenus sur l'île ${ }^{38}$. Une nouvelle facette de ces migrations réside dans le fait que les pays d'Afrique du Nord et du Moyen-Orient sont devenus eux-mêmes des pays de transit pour les migrants originaires d'Afrique sub-saharienne ${ }^{39}$.

Jusqu'à présent, peu d'études systématiques ont analysé la dimension méditerranéenne de la politique JAI, qui s'étend bien au-delà du simple aspect migratoire ${ }^{40}$. Pourtant, en ce qui concerne la CPJ dans la région euro-méditerra-

36. Expression utilisée par Anderson M. et Bort E. dans The Frontiers of the European Union, op. cit.

37. Baldwin-Edwards M., The Changing Mosaic of Mediterranean Migrations, Migration Policy Institute, 2004, disponible sur : http://www.migrationinformation.org/feature/display.cfm?ID=230

38. Balzan A., « MEP to make proposals to help Malta with immigration problem », EU Observer, Bruxelles, 30 mars 2006.

39. Pour une analyse de la dimension externe de la politique d'immigration voir : Parlement européen, Analyse de la dimension externe des politiques d'asile et d'immigration de l'UE-synthèse et recommandations pour le Parlement européen, Direction générale pour les politiques externes de l'Union, direction B, Bruxelles, 2006, p. 26.

40. A l'exception de Escheverrai Jesus C., La Cooperacion en asuntos de justicia e interior y el proceso de Barcelona: un balance, UNISCI Discussion Papers, UNISCI(2005), pp. 83-92; Lutterbeck D. « Policing Migration in the Mediterranean », Mediterranean Politics, vol. 11, n¹, 
néenne, la stratégie commune à l'égard de la Méditerranée, décidée lors du Conseil de Santa Maria de Feira en juin 2000, constitue la première étape identifiée dans la tentative d'injecter des éléments de politique JAI dans les relations euro-méditerranéennes, dans la droite lignée des objectifs décidés à Tampere en 1999. Le renforcement de la coopération dans le domaine JAI est affirmé comme objectif majeur, notamment dans les domaines de la lutte contre le crime organisé, le blanchiment d'argent et le trafic de drogue, à travers des programmes de formation des autorités judiciaires et des forces de police des pays partenaires. La collaboration est établie comme une priorité

« pour mettre en place le cadre juridique, institutionnel et judiciaire nécessaire pour poursuivre efficacement les auteurs de [ces] infractions et d'autre part, pour instaurer des mécanismes de coopération permettant de lutter contre la criminalité transfrontière 41 ».

A partir de 2001, l'agenda des diplomates en charge des questions politiques et sécuritaires, qui constituent le premier panier du Partenariat euro-méditerranéen (PEM), a été affecté progressivement par les réunions des ministres JAI. Si cela peut s'expliquer par des facteurs externes, comme les événements du 11 septembre, les facteurs internes ne doivent cependant pas être négligés. En effet, les discussions au sein du PEM étaient à l'époque dans l'impasse sur la question de la charte pour la paix et la stabilité du fait du conflit israélo-arabe, et avaient conduit les diplomates européens à chercher des coopérations sur les questions dites de «soft security 42 ».

Au cour de l'externalisation de l'agenda JAI envers la Méditerranée, réside le rôle joué par la présidence espagnole de l'UE en 2002, qui a œuvré pour créer des circonstances favorables à cet agenda. Ce dernier correspondait en effet aux priorités domestiques de l'Espagne et à sa lutte contre l'immigration clandestine et le trafic de drogue en provenance du Maroc, son voisin du Sud le plus proche.

«Le sommet de Valence a permis à l'Espagne, non sans beaucoup de difficultés en raison de l'absence du Liban et de la Syrie, mais aussi à cause du conflit au Moyen-Orient, de tenter d'introduire une dimension JAI dans les relations euro-méditerranéennes en discutant de la gestion commune des flux migratoires, de la lutte contre le trafic d'êtres humains et du renforcement de la coopération anti-terroriste ${ }^{43}$ ».

2006, pp. 59-82 ; Parlement européen, Analyse de la dimension externe des politiques d'asile et d'immigration de l'UE- synthèse et recommandations pour le Parlement européen, op. cit.

41. Conseil européen, Presidency Conclusions of Santa Maria Da Feira European Council - The Common Strategy of the European Union on the Mediterranean Region, Santa Maria Da Feira, Journal officiel des Communautés européennes, 2000/458/CFSP, 19 juin 2000.

42. Bicchi F., From Security to Economy and Back? Euro-Mediterranean Relations in Perspective, European University Institute, pp. 7-8.

43. Arteaga F., "The balance of the Spanish Presidency of 2002 with regard to Justice and Home Affairs of the European Union ", The Spanish Presidency of the EU, University of Liverpool, 2002 
A cette époque, l'Espagne se trouvait dans une position exceptionnelle pour influencer le cours des négociations puisque, comme l'a souligné Gillespie, la relation Aznar-Blair s'était trouvée renforcée par la lutte contre le terrorisme ( «fight against terror»), et avait gagné en marge de manœuvre alors même que l'alliance franco-allemande ne progressait que lentement en raison de contraintes électorales internes ${ }^{44}$.

Le Document d'encadrement de Valence constitue le premier programme multilatéral jamais conclu sur les questions de JAI dans les relations euro-méditerranéennes. Le "Programme de co-opération régionale dans le domaine de la justice, la lutte contre la drogue, le crime organisé et le terrorisme, ainsi que la coopération dans le traitement des questions liées à l'intégration sociale des migrants, de la migration et des mouvements de personnes 45 » devait donner un nouvel élan au PEM 46. Il est intéressant de noter que, exceptionnellement, le document a été conclu dans un premier temps au niveau des hauts fonctionnaires, grâce à la stratégie espagnole de conclure d'abord un accord avec les partenaires méditerranéens, et ensuite de trouver un consensus dans l'UE, une initiative différente des pratiques diplomatiques traditionnelles au sein du PEM ${ }^{47}$. Par conséquent, l'UE a organisé en juin 2003 un séminaire «MEDA-Justice et Affaires intérieures » durant lequel la Commission a décidé de conclure un contrat de 2 millions d'euros pour chaque section du programme (migration, police et justice, sous la houlette du programme MEDA/JAI I pour un montant de 6 millions d'euros) ${ }^{48}$. Cette coopération JAI a été présentée comme faisant partie d'un processus plus large de revitalisation des

" gouvernements et du développement de la démocratie, des droits de l'Homme et de l'état de droit, qui devra considérablement améliorer l'attraction de la Méditerranée pour les investisseurs et aider à rapprocher les partenaires méditerranéens de l'UE 49 ».

En 2005, une seconde enveloppe MEDA/JAI a été proposée, pour un montant de 15 millions d'euros sur la période 2005-2006. Le « Programme régional indicatif pour 2005-2006 » réitère l'importance de l'élément JAI dans la politique méditerranéenne de l'UE et identifie des domaines prioritaires pour la CPJ : le

44. Quelques mois avant le sommet de Valence, la France vivait une élection présidentielle exceptionnelle, avec la présence de Jean-Marie le Pen au second tour. Voir Gillespie R., "Reshaping the agenda? The internal politics of the Barcelona process in the aftermath of September 11 ", in Jünemann A., Euro-Mediterranean Relations after September 11. International, Regional, Domestic Dynamics, London, Franck Cass, 2004, pp. 21-35.

45. Conférence euro-méditerranéenne, Presidency Conclusions - Valencia Action Plan, $V^{\text {th }}$ Euro-Mediterranean Conference of Foreign Ministers, Valencia, 2002.

46. Tel que nous l'a confié un fonctionnaire de la DG JLS, le long titre du document de Valence reflète la sensitivité affichée à l'époque par les partenaires méditerranéens quant à la présence explicite du terme « Justice et Affaires Intérieures ».

47. Gillespie R., op. cit., p. 26.

48 . Voir l'annexe 4 de ce numéro.

49. Commission européenne, "Euro-Mediterranean Partnership and MEDA Regional Activities ", Euromed Information Notes, 2005, p. 115 et p. 30. 
contrôle des frontières, la gestion des flux migratoires, la lutte contre le terrorisme, le blanchiment d'argent, la promotion d'un système judiciaire indépendant, ainsi que la coopération judiciaire en matières pénale et civile, le droit de la famille étant inclus ${ }^{50}$. A l'heure actuelle, les services de la Commission européenne planchent sur le programme MEDA/JAI III qui sera mis en place pour la période 20082013. Il est envisagé que les agences Frontex et Europol deviennent gestionnaires de certains projets ${ }^{51}$. Entre 2003 et 2006, l'enveloppe financière consacrée au programme MEDA/JAI a été multiplié par 2,5 fois. Au total, depuis le lancement du « Document d'encadrement » de Valence en 2002, environ 155 millions d'euros ont été employés à la réalisation de la coopération JAI en Méditerranée, à travers les programmes régionaux MEDA/JAI I (6 millions d'euros), MEDA/JAI II (15 millions d'euros), ainsi qu'au niveau bilatéral avec une enveloppe avoisinant les 134,2 millions d'euros. A cela s'ajoutent également les programmes de coopération bilatéraux entre les partenaires méditerranéens et les Etats-membres de l'UE.

Le futur MEDA/JAI III devra être appliqué dans le cadre de l'instrument européen de voisinage et de partenariat (IEVP) et de la PEV, qui constituent désormais le cadre des négociations avec les voisins méditerranéens, mais aussi celui de l'institutionnalisation de la coopération policière et judiciaire.

\section{La PEV : vers l'institutionnalisation de la coopération policière et judi- ciaire euro-méditerranéenne?}

\section{La PEV ou l'institutionnalisation de la politique JAI}

Le développement de la PEV ces dernières années reflète l'externalisation des questions JAI vers la PEE, notamment en ce qui concerne la gestion des « nouveaux » et «anciens » voisins. Documents après documents, la PEV a évolué d'une approche plutôt généreuse envers les voisins de l'UE à une position qui peut se résumer par la formule «tout sauf les institutions » ${ }^{52}$, et qui est marquée par un retour au bilatéralisme. Cet article défend l'argument selon lequel la PEV a intensifié l'institutionnalisation des questions JAI au cœur de la relation euro-

50. Commission européenne et EuroMed, Regional and Bilateral MEDA Co-operation in the Area of Justice, Freedom and Security, Information Note, 2003, p. 5.

51 . Entretien confidentiel, DG Relex, Bruxelles, 20 avril 2006.

52. Il est important ici de souligner que la Commission européenne a rapidement évolué du concept d' «Europe élargie » («Wider Europe ») vers le terme de « Nouvelle Politique de voisinage » («New Neighbourbood Policy»), et a finalement opté pour la « Politique européenne de voisinage » («European Neighbourhood Policy ). Ces évolutions ont été accompagnées de changements du contenu de cette politique mais également de son étendue géographique. Ainsi alors que la Communication de 2003 sur Wider Europe se voulait généreuse et promettait une coopération proche de l'Espace économique européen, fondée sur l'accès aux quatre libertés (liberté de circulation des personnes, des biens, des services et des capitaux), très vite ces promesses initiales ont disparu de la Communication suivante de 2004. Voir aussi Comelli M., The European Neighbourhood Policy (ENP): Distinctive Features and Differences with the EuroMediterranean Partnership, IGC Net conference, Brussels, 2005, disponible sur : http://www.igc-net.org/Conference_17Nov05/Comelli_ENP_Barcelona_Dec05.pdf 
méditerranéenne. Une analyse des plans d'actions de la PEV révèle en effet une clarification des questions JAI en général, et de la CPJ plus spécifiquement ${ }^{53}$.

Au cœur de la PEV réside le principe de différentiation qui consiste à accompagner les réformes des pays voisins en les « récompensant » au cas par cas, selon les progrès réalisés. Ce principe est également appliqué pour la réalisation de projets JAI, par exemple avec le Maroc, la Jordanie et l'Algérie qui se sont vus attribuer des fonds pour «les programmes soutenant l'application des Accords d'Association ${ }^{54}$. Ces programmes spécifiques sont des :

«actions d'alignement avec les voisins qui sont les mieux préparés dans les domaines prioritaires : contrôle des frontières et gestion des flux migratoires, lutte contre le financement des activités terroristes et du blanchiment d'argent, réformes judiciaires et juridiques liées aux aspects fondamentaux de la justice (accès à une justice indépendante et impartiale), particulièrement dans ses effets sur la coopération judiciaires en matières pénales et civile, incluant le domaine de droit de la famille; du droit du commerce et de la coopération judiciaire criminelle $55 »$.

Pour atteindre ces objectifs, l'UE a recours à des instruments spécifiques tels que les «projets de jumelages» de dimension régionale, mais également entre Cours suprêmes des deux rives, l'établissement de "protocoles d'accord $\$ 56$ entre les offices spécialisés de plusieurs pays pour organiser l'échange d'information sur le blanchiment d'argent, et enfin «l'échange de bonnes pratiques» sur la lutte contre l'immigration clandestine.

Le but ultime de ces programmes étant l'institutionnalisation de la CPJ dans la région, l'UE souhaite que «la multiplication d'actions dans un format spécifique puisse donner lieu à des ensembles intra-régionaux dans le domaine JAI ». Le Programme indicatif régional (PIR) 2005-2006 évoque ainsi la possibilité de créer un Centre euromed des hautes études judiciaires concernant les fondamentaux de la justice, un centre interrégional euromed de coopération policière pour le développement des coopérations techniques entre services de police, ou bien encore la création d'un centre euromed d'études des migrations pour la mise à jour et la mise en commun des données pluridisciplinaires requises par la question migratoire. De tels objectifs s'inscrivent dans l'ambition normative de l'UE de créer un «partenariat régional sécuritaire » avec ses voisins du Sud. Cet objectif est affiché dans le Document de stratégie régionale (DSR) et dans le PIR pour

53. Voir l'annexe 6 de ce numéro.

54. European Commission and EuroMed, Regional and Bilateral MEDA Co-operation in the Area of Justice, Freedom and Security, p. 11.

55 . European Commission, MEDA, Regional Indicative Programme 2005-2006, 2005, disponible sur : http://ec.europa.eu/comm/external_relations/euromed/rsp/nip0506.htm

56 . Dans le texte original «memorandum of understanding ». 
la période 2007-2013, qui ont érigé comme priorité principale «la création d'un espace euro-méditerranéen commun de coopération en matière de justice, de sécurité et de migration", devant l'objectif d'un espace économique durable et de celui de l'établissement d'une sphère commune d'échanges socioculturels ${ }^{57}$. Le document met en avant les «mesures de confiance » sur lesquelles repose la coopération JAI, à savoir la coopération en matière de protection civile, ainsi que le « Partenariat pour la paix » pour la résolution du conflit israélo-palestinien qui se retrouve désormais dans le chapitre "Coopération en matière de politique, de justice, de sécurité et de migration ». Parallèlement, dans l'esprit de la PEV, l'UE veut promouvoir la «coopération Sud-Sud», afin de pouvoir décentraliser à son "proche étranger» la gestion des menaces. Mais il est évident que le partage des tâches («burden-sharing ») ne se réalisera pas sans partage des responsabilités ( «esponsibility-sharing »), à travers une réelle inclusion des partenaires méditerranéens dans la définition d’un agenda JAI commun ${ }^{58}$.

A l'heure actuelle, tous les partenaires du processus de Barcelone ont conclu un plan d'action pour la PEV, à l'exception de l'Algérie, de la Syrie et de la Libye. Le plan d'action de l'Egypte est le dernier en date, après de longues tractations qui ont finalement mené à sa signature en mars 2007. La procédure veut que la Commission européenne prépare un rapport sur le pays en question, dans lequel elle passe en revue sa situation politique, économique et sociale et identifie les priorités pour de futures actions. Des discussions ont lieu avec les partenaires ainsi qu'avec les présidences de l'UE, le secrétariat du Conseil et le bureau du Haut Représentant pour la PESC. Les plans d'actions sont alors envoyés au Parlement européen, au Comité économique et social, ainsi qu'au Comité des régions 59 . Pour les partenaires méditerranéens, la base légale demeure celle des accords d'association euro-méditerranéens. Le conseil d'association et ses sous-comités sont en charge d'exécuter et de contrôler l'application des plans d'actions. Ainsi, les sous-comités «Justice et Sécurité », crées à l'occasion des accords d'association, permettent de mettre en œuvre la coopération en matière JAI. Dans le cas du Maroc, ce sous-comité existe depuis février 200360.

Bien que la CPJ ne soit pas une nouveauté en Méditerranée, du fait de la coopération qui existait déjà entre Etats, celle-ci se retrouve néanmoins pour la première fois en haut de l'agenda politique euro-méditerranéen. Elle est également institutionnalisée dans le cadre de la PEV à travers son plan d'action et ses sous-comités. Cette institutionnalisation renforce l'aspect bilatéral des relations en matière JAI, comme le démontre le cas du Maroc.

57. Commission européenne, Instrument européen de voisinage et de partenariat (IEVP), Document de stratégie régionale (2007-2013) et Programme indicatif régional (2007-2013) pour le partenariat euro-méditerranéen, Bruxelles, 2007, p. 3 et p. 60.

58 . Voir l'annexe 3 de ce numéro sur les objectifs de CPJ en Méditerranée.

59. Commission européenne, Communication from the Commission to the Council on the Commission Proposals for Action Plans under the ENP, 2004, p. 6.

60. Entretien confidentiel, Mission du Maroc auprès de l'UE, 7 février 2006. 
La Maroc a été parmi les premiers pays à accueillir favorablement la PEV et son plan d'action, car celui-ci correspond au programme national de réforme du Royaume ${ }^{61}$. Cet agenda s'est concrétisé en 2003 par un nouveau code de la famille («Moudawana»), qui a notamment fait évoluer le statut des femmes ${ }^{62}$. Un autre exemple concerne l'initiative du roi d'établir, en janvier 2004, une Commission équité et réconciliation qui a exploré les cas de disparitions et de détentions sous le règne de son père le roi Hassan II ${ }^{63}$. Il faut également noter que le Maroc demeure le seul pays arabe ayant officiellement demandé l'adhésion à l'UE en juillet 1987.

Le Maroc a fait partie de la première vague des pays méditerranéens, avec la Tunisie, l'Autorité palestinienne et Israël, pour lesquels les accords d'association euro-méditerranéens sont entrés en vigueur dans les cinq années ayant suivi le processus de Barcelone. C'est également avec la même rapidité que le plan d'action de la PEV pour le Maroc a été adopté par l'UE en février 2005, et par le Maroc en juillet de la même année. Le plan d'action énumère les opérations à mettre en œuvre afin de "poursuivre le développement de la coopération entre les autorités judiciaires et de police du Maroc et des Etats-membres». Cette coopération passe notamment par un état des lieux de la coopération judiciaire entre le Maroc et l'UE, l'inventaire des bonnes pratiques et la négociation d'un accord de coopération entre le Maroc et Europol.

Selon un diplomate européen, le Maroc a été un partenaire avec lequel il fut aisé de négocier, étant donné les accords qui existaient déjà en matière de migration ${ }^{64}$. En 1999, le Conseil JAI, désireux d'aborder la question des migrations d'un point de vue horizontal, a demandé au Groupe de haut niveau « Asile et Migration » de préparer un plan d'action pour l'Afghanistan, le Maroc, l'Irak, la Somalie et le Sri Lanka. L'Espagne a été désignée coordinatrice du plan d'action, sa coopération avec le Maroc étant très étroite. Ce plan d'action a notamment conseillé la création d'un sous-comité sur les questions migratoires dans le cadre du PEM 65. Par ailleurs, il faut souligner que la CPJ avait déjà été initiée dans divers forums. Dans les années 1990, les Etats-membres ont commencé à ouvrir leurs propres groupes de coopération au Maroc. Ainsi, dès 1999, la gendarmerie royale marocaine a rejoint

61. Le rapport souligne en effet que le Maroc note avec satisfaction la possibilité d'une approche bilatérale et différenciée basée sur la volonté politique et la capacité concrète de chacun des partenaires en vue de faire évoluer l'association vers une relation qui correspond à la situation spécifique de chaque pays. Commission Européenne, Document de travail de la Commission, Politique européenne de voisinage, Rapport sur le pays, Maroc, COM (2004) 373, Bruxelles, 12 mai 2004.

62 . Les femmes marocaines ne sont désormais plus légalement liées aux décisions de leurs parents masculins en ce qui concerne le mariage, l'éducation et leur vie professionnelle.

63. Carnegie Endowment for International Peace and FRIDE Morocco-Carnegie Endowment Report.

64 . Entretien confidentiel, DG JLS, Bruxelles, 7 février 2006.

65. Council Document 11426/99 JAI 75 AG 30, 30 septembre 1999. 
l'association des forces de police et de gendarmerie européennes et méditerranéennes à statut militaire, la FIEP, acronyme pour « France-Italie-Espagne-Portugal », qui en ont été les membres fondateurs en 199466.

Etant donné sa position géographique spécifique, le Maroc revêt un intérêt stratégique majeur pour l'UE afin de renforcer la CPJ dans le domaine des migrations et du terrorisme. Au carrefour de plusieurs grandes routes de migration, le Maroc est en effet devenu un pays de transit pour l'immigration subsaharienne. Les événements dramatiques de Ceuta et Melilla en 2005 ont accéléré la prise de conscience du côté marocain qu'il était nécessaire que le gouvernement s'implique davantage dans la gestion des migrations. Le Maroc a ainsi été à l'origine de la conférence euro-africaine «Migrations et Développement » sur les questions migratoires avec la France et l'Espagne ${ }^{67}$ en juillet 2006. Sur le plan du terrorisme, ces trois pays ont proposé la constitution d'un réseau de CPJ euroméditerranéen contre le terrorisme, le trafic de drogue et le trafic d'êtres humains. Les orientations ont été présentées par le ministre de l'Intérieur espagnol José Antonio Alonso lors du meeting informel des ministres JAI à Newcastle, en Grande-Bretagne en septembre $2005{ }^{68}$. Des demandes ont également été formulées par le gouvernement marocain afin de bénéficier de l'assistance de l'UE pour mettre en place une «Financial Intelligence Unit» afin de prévenir le financement des activités de terrorisme ${ }^{69}$. Sous la houlette du programme MEDA/JAI, les ministères de l'Intérieur français et marocain ont également signé le premier accord de jumelage dans le domaine JAI en décembre 2005. Avec un budget de 20 millions d'euros, ce programme se concentre sur la migration et la surveillance des frontières 70 .

\section{L'externalisation de JAI : quelles révélations pour l'UE en tant qu'acteur de politique étrangère?}

L'externalisation de la politique JAI et son institutionnalisation dans les relations euro-méditerranéennes, notamment à travers la CPJ et la PEV, est donc un phénomène confirmé. Il s'agit désormais d'en interpréter les implications pour l'UE en tant qu'acteur de relations internationales. A la lumière du développement de la dimension extérieure JAI, il est pertinent de s'interroger si ce phénomène reflète des attributs d' « actorness » de l'UE, et témoigne de l'aptitude de l'UE à "capitaliser sur sa [...] présence, et donc de son fonctionnement effectif en tant qu'acteur global 71 ». Se référant à la littérature sur

66. Voir http://www.fiep-asso.org/

67. Entretien confidentiel, mission du Maroc auprès de l’UE, 7 février 2006.

68. Magharebia agency, 11 septembre 2005.

69. Vries G.D., Introductory Speech, Séminaire organisé par la présidence de l'UE sur la prévention du financement du terrorisme, Bruxelles, 22 septembre 2004.

70. Synopsis Euromed, Lettre d'information hebdomadaire sur le Partenariat Euro-Méditerranéen et le Programme MEDA, n³41, 12 janvier 2006.

71 . Bretherton C., Vogler J., The European Union as a Global Actor, Oxon, Routledge. 2006, p. 218. 
l'« actorness » de l'UE, notre analyse distingue des implications à la fois pour la légitimité de l'action de l'UE dans ses relations extérieures, mais également des implications au niveau interne.

\section{Le concept d'« actorness»}

Au cours des années 1990, des universitaires prudents préféraient utiliser le terme de "présence » de l'UE, plutôt que d' "actorness » en termes de relations internationales ${ }^{72}$, où le rôle et la présence de l'UE ne se trouvent pas toujours de manière simultanée. Au contraire, l'UE oscille entre «présence » et « actorness » selon les différentes politiques qui constituent son action extérieure. Par " présence », Allen et Smith se sont à l'époque référés à «la capacité d'exercer une influence, de modeler les perceptions et les attentes des autres 73 ». Pour d'autres, comme Vogler, le concept de "présence » constitue simplement l'une des multiples facettes de l'actorité de l'UE ; cette dernière devant être conçue comme un processus évoluant autour de trois concepts : présence, opportunité et capacité. Le terme "présence » se réfère aux perceptions et aux attentes des autres acteurs internationaux par rapport à l'UE. «Opportunité » désigne les facteurs de l'environnement extérieur «qui permettent ou contraignent son action». Enfin, « capacité» se rapporte à «l'babilité de formuler et de mettre en ceuvre une politique extérieure, à la fois en développant un agenda politique proactif et en répondant efficacement aux attentes extérieures, aux demandes et aux opportunités » ${ }^{4}$. Par ailleurs, «l'actorness » de l'UE repose sur cinq éléments clés : un engagement envers des valeurs et des principes communs, la capacité de définir des priorités politiques de manière cohérente, la capacité de mener des négociations internationales, l'accès et la capacité à utiliser des instruments politiques, et enfin la légitimité du processus de décision ${ }^{75}$.

Dans notre étude de cas, trois obstacles majeurs se posent à l'UE en tant qu'acteur de relations internationales. Au niveau externe, il est important de souligner les risques de mener simultanément des objectifs contradictoires dans

72 . Hill C., The Actor's of Europe's Foreign Policy, Londres, Routledge, 1996; Bretherton C., Vogler J., The European Union as a Global Actor, Londres, 1999 ; Ginsberg R., The European Union in International Politics: Baptism by Fire, Lanham, MD, Rowman and Littlefield, 2001 ; Smith H., European Union Foreign Policy: What is its and what it does, Londres, Pluto Press, 2002 ; Knodt M., Princen S., « Understanding the EU's external relations. The move from actors to processes ", in Understanding the EU's external relations, Londres, Routledge, 2003, pp. 195208 ; Smith K., European Union Foreign Policy in a Changing World, Londres, Pluto Press, 2003.

73. Nous traduisons la citation. Cité dans Biscop S., «Effective multilateralism and collective security: empowering the UN », Haddadi S., " Political securitisation and democratisation in the Maghreb: ambiguous discourses and fine-tuning practices for a security partnership », Working Paper IES Berkeley, n²3, 2004, p. 7 et p. 26. IIEB Working Papers, Institute for International and European Policy, Leuven, Katholieke Universiteit Leuven, 2005, p. 22.

74. Vogler J., In the Absence of the Hegemon: EU Actorness and the Global Climate Change Regime, The European Union in International Affairs, National Europe Centre, Australian National University, 2002, p. 6.

75 . Bretherton C., Vogler J., The European Union as a Global Actor, Oxon, Routledge, 2006 p. 30. 
la région méditerranéenne. Ensuite, au niveau interne, se pose le problème de cohérence qui découle de la «pillarisation » du processus décisionnel et de la présence de plusieurs acteurs institutionnels qui défendent des visions différentes de la sécurité européenne.

\section{Des objectifs concurrents de politique étrangère}

Notre analyse a démontré à quel point la CPJ avec les pays méditerranéens, le Maroc en particulier, a connu une réelle accélération. La question sous-jacente est de savoir si l'export d'un modèle de politique JAI dans les relations de l'UE avec ses partenaires méditerranéens influencera positivement ou non les réformes dans ces pays. En effet, le risque est qu'un accroissement de la CPJ avec les pays méditerranéens soit manipulé par les gouvernements autocrates en place. Dans cette hypothèse, ces derniers utiliseraient la coopération antiterroriste pour justifier la violence exercée à l'encontre de l'opposition politique, bien souvent islamiste. C'est ici que se pose la question de la cohérence entre les objectifs de la dimension extérieure JAI et le reste des objectifs de politique étrangère de l'UE, notamment en termes de réformes démocratiques et de promotion de l'Etat de droit. Cette situation réveille en effet les vieux démons du dilemme sécuritisation vs. démocratisation; l'UE étant prise au piège entre ses ambitions normatives dans la région et ses peurs de voir les islamistes arriver au pouvoir, et ainsi prendre en otage la démocratie ${ }^{76}$. Le cas de la victoire du Hamas, suite à un processus électoral transparent, qui respectait les critères démocratiques mis en avant par l'UE, a mis en lumière les paradoxes de l'action de l'UE. En suspendant son aide au Hamas, l'UE a contribué à l'affaiblissement de la gouvernance démocratique en Palestine. Par ailleurs, il faut souligner que, depuis 2001-2002, les programmes de promotion de l'Etat de droit ont été progressivement transférés du chapitre « démocratisation » vers les chapitres de coopération JAI, soulignant ainsi la tendance à sécuritiser les efforts de démocratisation.

En insistant sur le principe de différentiation dans le domaine sécuritaire, l'UE doit être consciente que la compétition entre les partenaires méditerranéens risque d'entraver la diffusion de l'Etat de droit et des libertés fondamentales. Il serait en effet dangereux de jouer au « jeu de la carotte et du bâton » dans le domaine de la coopération sécuritaire. Au contraire, il est fondamental que la CPJ soit accompagnée de programmes appropriés qui renforcent la capacité administrative des institutions judiciaires et policières. C'est pourquoi des efforts financiers, mais également humains, doivent être dévolus à la formation des agences judiciaires et policières, qui ne sont pas indépendantes des régimes autoritaires méditerranéens. Cela permettrait d'assurer que l'externalisation des questions

76. Haddadi S., « Political securitisation and democratisation in the Maghreb: ambiguous discourses and fine-tuning practices for a security partnership ", Working Paper IES Berkeley, n²3, 2004, p. 7 et p. 26. 
JAI ne vienne pas contrarier les efforts de l'UE en matière de démocratisation, et d'éviter que cela ne conforte dans leur pouvoir les mamelukes contemporains.

\section{(In)cohérence interne : institutions et politiques}

Habituellement, l'UE déploie son action extérieure à travers la PESC et la PESD, mais également à travers ses actions dans les domaines de politique du développement, du commerce et de l'humanitaire. Différents acteurs et modes de décision sont donc impliqués dans la quête d'«actorness " de l'UE. Différentes opportunités sont contraintes par différents types de modes de décision. Par conséquent, il n'existe pas de cadre institutionnel unique pour l'action extérieure de l'UE. Au contraire, «les règles du jeu des différents domaines politiques de relations extérieures varient considérablement 77 » et pèsent sur la cohérence de l'action extérieure de l'UE.

Quand bien même une partie des questions JAI ont été communautarisées, le Conseil européen et le Conseil des ministres demeurent les acteurs clés du processus décisionnel, en fournissant les orientations politiques pour l'intégration des questions JAI dans l'action extérieure de l'UE ${ }^{78}$. Dans un rapport clé du COREPER daté de 2000, le lien a été établi entre les préoccupations de sécurité internes et la PESC. Le rapport souligne la nécessité pour l'UE d'intégrer les questions JAI dans sa politique extérieure, afin que des actions compréhensives, intégrées et horizontales soient mises en œuvre par l'UE 79 . Ce rapport révèle la volonté du COREPER et du Conseil de garder la main sur un processus d'externalisation des questions JAI. Les groupes influents du Conseil tels que le Comité de l'article 36 (CATS), le Comité stratégique sur l'immigration, les frontières et l'asile (SCIFA), ainsi que le Comité droit civil sont ainsi impliqués dans le processus décisionnel en évaluant et orientant le développement de la dimension extérieure JAI.

En ce qui concerne l'influence de la Commission européenne, il est légitime de se demander dans quelle mesure ce développement a renforcé la marge de manœuvre de cette institution quant à l'action extérieure de l'UE, dont elle est traditionnellement exclue, du fait du processus intergouvernemental de la PESC. L'analyse de la dimension extérieure de la politique JAI démontre que le Conseil et la Commission européenne jouent tous deux le rôle «d'entrepreneurs politiques ». Les présidences successives de l'UE ont en effet lancé un

77. Vanhoonacker S., « The institutional framework », in Hill C., Smith M., International Relations and the European Union, Oxford, Oxford Press University, 2005, pp. 67-89, voir p. 68.

78. Ce phénomène reflète la compétition croissante entre les ministres des Affaires étrangères et les ministres de l'Intérieur au niveau des Etats-membres, comme a pu l'illustrer l'affrontement entre Dominique de Villepin, alors ministre des Affaires étrangères, et le ministre de l'Intérieur de l'époque, Nicolas Sarkozy.

79. COREPER, Note point "A" au Conseil affaires générales / Conseil européen "Priorité et objectifs de l'UE pour les relations extérieures dans le domaine de la Justice et des Affaires intérieures », 7653/00, JAI 35, Bruxelles, 6 juin 2000. 
mouvement de "JAIsation » de l'action extérieure de l'UE à travers une série de programmes multi-présidences ${ }^{80}$. Depuis 2004, la Commission a renforcé son action dans le domaine JAI, bien que l'héritage historique de l'intergouvernementalisme demeure fort dans ce domaine. L'apogée de cette politique d'entreprenariat est illustrée par la PEV qui implique à la fois le Conseil et la Commission dans la rédaction et dans les négociations avec les pays voisins.

Du point de vue du processus décisionnel, l'externalisation des questions JAI conduit à l'augmentation des contraintes pesant sur l'action extérieure de l'UE. La politique JAI se caractérise en effet par un processus décisionnel complexe et multipiliers, qui reflète le compromis entre la coopération transgouvernementale et la communautarisation incrémentale ${ }^{81}$. Dans l'esprit des négociateurs du traité de Maastricht, la politique JAI et la PESC avaient été conçues comme deux politiques séparées, qui évolueraient dans deux piliers distincts, avec un fort élément intergouvernemental et très peu de marge de manœuvre pour les institutions européennes. La Commission, à l'époque, ne disposait que d'un droit d'initiative partagé en matière JAI (article K du traité sur l'Union européenne) et a dû subir plusieurs contraintes constitutionnelles, notamment à travers des instruments tels que les actions communes et les positions communes, héritage de la prise de décision intergouvernementale ${ }^{82}$. En 1997, cependant, un changement a eu lieu avec le transfert des questions migratoires, d'asile et des visas vers le premier pilier, ainsi que le contrôle des frontières. L'unanimité au Conseil JAI devait rester pour une période transitoire de cinq ans, qui s'est achevée en 2005, lorsque ces questions ont été transférées au premier pilier, et le processus de co-décision étendu à ces domaines. Selon une logique néo-fonctionnaliste, cette évolution devrait à terme rehausser le rôle de la Commission et du Parlement européen, mais également assurer à la Cour européenne de justice la possibilité d'examiner la légalité des décisions JAI.

Toutefois, la structure multi-piliers et les interactions entre politique JAI, PESC et PESD créent des problèmes additionnels à la cohérence de l'action de l'UE. Ainsi, en matière de migration si le cadre communautaire prévaut, pour ce qui est de la CPJ en matière de terrorisme il s'agit d'un processus intergouvernemental. La coopération avec les pays tiers est donc l'objet de multiples modes de gouvernance qui contribuent à l'accroissement de la complexité.

Par ailleurs, il est vrai que l'externalisation de la politique JAI habilite les institutions européennes à agir dans de nouveaux domaines de compétence, offrant un nouveau levier à l'UE sur la scène internationale. Ainsi, à la frontière entre les domaines de la PESC et de la PESD, les missions de police de l'UE se

80. Par exemple : Conseil européen, «JHA external relations multi-presidency programme», JAI 255, RELEX 317, The Netherlands Presidency, Brussels, 11044/04, 2004.

81. Lavenex S., W. Wallace, «Justice and Home Affairs », op. cit.

82. Pour une bonne analyse de ces contraintes voir : Uçuarer E., «From the sidelines to center stage: sidekick no more? The European Commission in Justice and Home Affairs ", European Integration Papers Online (EIOP), vol. 5, 2001. 
sont considérablement développées depuis quelques années, permettant ainsi aux experts JAI d'investir le domaine des missions civiles de l'UE ${ }^{83}$. Les experts JAI et leurs comités sont impliqués dans la définition des rôles et des tâches que les forces de police doivent mener ${ }^{84}$. En novembre 2005, le Conseil, a établi, à travers une action commune, une mission de police dans les territoires palestiniens. Le principal objectif de la mission EUPOL COPPS est d'améliorer les capacités policières et judiciaires de l'Autorité palestinienne. A cette fin, la mission assiste la police palestinienne civile dans l'application du Programme de développement des forces de police, et travaille de près avec les hauts fonctionnaires palestiniens. La mission coordonne l'assistance de l'UE et celle des Etatsmembres, et fournit des conseils sur les éléments de justice pénale.

Cependant, au-delà du défi institutionnel, il s'agit pour les institutions européennes de trouver un équilibre entre deux traditions sécuritaires : la tradition inclusive des diplomates, et la tradition dite « exclusive » des ministères européens de l'Intérieur.

\section{Perceptions sécuritaires : des valeurs partagées?}

L'externalisation des questions JAI n'est pas sans influence sur les perceptions et les cadres cognitifs. En effet, le lien entre inclusion et exclusion permet de mieux comprendre deux traditions de la culture européenne sécuritaire. La référence à la métaphore du Prince de Machiavel illustre ce point ${ }^{85}$. L'externalisation des questions JAI révèle deux visions opposées de la gouvernance européenne. D'un côté les diplomates européens ont longtemps favorisé une culture de la sécurité selon laquelle le développement des questions sociales, économiques et environnementales doit faire partie intégrante de l'action extérieure de l'UE. Il s'agit d'une vision inclusive, c'est-à-dire qui souhaite intégrer le proche étranger dans les politiques communautaires, afin de partager avec eux les bénéfices du marché commun, et notamment ses quatre libertés fondamentales de libre circulation. Les expériences successives de partenariats régionaux et des élargissements ont progressivement conduit à une inclusion des voisins de l'UE (par exemple, les anciens membres de l'Association européenne de libre échange et les accords européens signés avec les pays d'Europe centrale et orientale).

Parallèlement, une culture dite "exclusive » caractérise les ministères de l'Intérieur européens, ainsi que les experts JAI. Cette vision insiste sur la consolidation de l'espace de liberté, sécurité et justice pour les citoyens européens qui passe par la consolidation des frontières extérieures de l’UE. Cette

83. Voir par exemple l'analyse sur ce sujet de Mounier G., « European Police Missions: From Security Sector Reform to Externalization of Internal Security beyond the Borders », op. cit. 84 . Rees W. «The external face of internal security », op. cit., p. 222.

85. Pastore J., op. cit. 
vision se trouve aux antipodes de celle des diplomates et peut expliquer les incohérences qui caractérisent l'action extérieure de l'UE vis-à-vis de ses voisins. Ainsi, en Méditerranée, l'UE est prise entre son souhait de favoriser l'accès de ses voisins à certaines politiques et programmes communautaires, alors même qu'elle insiste sur la CPJ, et donc sur la nécessité pour les partenaires méditerranéens de participer à la consolidation des frontières de l'Europe et à leur imperméabilité en les incluant dans la gestion des menaces.

Au contraire, les efforts diplomatiques ont conduit à des accords régionaux avec les voisins de l'UE, de la Russie à la Méditerranée, en passant par les Balkans, afin de construire une communauté de valeurs, qui pourrait mener à la création d'une communauté de sécurité régionale. Dans se sens, le PEM constitue un "rêve constructiviste 86 » selon lequel à travers la persuasion active et la socialisation, un sentiment de « vouloir vivre » ensemble émergerait. Le premier pas vers la création d'une communauté euro-méditerranéenne a d'ailleurs été de développer un partenariat non seulement politique et économique, mais également social et culturel, afin de consolider les bases d'un dialogue entre civilisations.

Etant donné l'importance grandissante des question JAI, à la fois au niveau européen et au niveau des Etats-membres, les efforts diplomatiques se retrouvent confrontés à l'émergence du « discours de sécuritisation » des experts JAI, pour emprunter le concept de Buzan ${ }^{87}$. Les deux discours concurrents décrits plus haut, sont désormais réunis sous un même cadre politique : la PEV.

Le développement de la dimension extérieure de la JAI a considérablement gagné en portée et en contenu depuis ses débuts en 1999. Comme l'ont justement souligné Lavenex et Wallace, l'ambition de créer un « espace de liberté, sécurité, et justice » (ELSJ) est comparable à celle qui existait lors de la création du marché unique, bien que les questions JAI soient davantage liées aux questions de souveraineté ${ }^{88}$. Cette ambition s'est développée parallèlement aux relations extérieures et porte désormais l'ambition de créer un ELSJ avec ses voisins.

Cet article a tenté d'offrir un panorama de la dimension extérieure de la politique JAI et de ce que ce concept recouvre dans le cadre des relations euroméditerranéennes. Inscrit dans un contexte de perceptions sur la sécurité européenne qui ne cessent d'évoluer, où les frontières s'estompent de plus en

86. Crawford B., Why the Euro-Med Partnership? Explaining the EU's Strategies in the Mediterranean Region, Institue of European Studies, UC Berkeley, p. 13.

87. Pour « l'école de Copenhague ", les menaces sécuritaires n'existent pas en tant que telles, mais sont le résultat de mécanismes discursifs par lesquels les autorités publiques insistent sur l'urgence de ces questions et la nécessité de les adresser en dehors du processus politique traditionnel à travers des mesures exceptionnelles. Voir Buzan B., Waever O., de Wilde J., Security: a new framework for analysis, Lynne Rienner, London, 1998, $239 \mathrm{p}$.

88 . Lavenex S., Wallace W., «Justice and Home Affairs », op. cit., p. 458. 
plus, la dimension extérieure de la politique JAI est devenue un objectif de politique étrangère. Non seulement incluse de manière systématique dans les politiques extérieures de l'UE, la politique JAI a aussi été intégrée dans le cadre de la PEV qui est devenue une priorité centrale dans les relations de l'UE avec ses voisins. Cette évolution n'est pas sans induire des problèmes pour la cohérence externe de la politique étrangère de l'UE qui comporte désormais des objectifs de politique étrangère concurrents, mais également pour sa cohérence interne, du point de vue des acteurs et du processus décisionnel. La multiplication des acteurs en charge de cette politique et le fait que celle-ci soit régie par différentes règles, selon les piliers communautaires ajoutent une certaine dose de complexité au processus politique et reflète au grand jour le dilemme qui existe entre les cultures sécuritaires inclusives et exclusives.

L'agenda de recherche de la dimension extérieure JAI doit amener les universitaires qui se penchent sur ce phénomène à analyser les dynamiques sousjacentes qui existent dans les trois piliers et favoriser une approche dite de "cross-pillarization », qui fasse tomber les barrières entre l'étude différenciée de la politique JAI et de la PEE. Les axes d'investigation futurs pourront se concentrer sur les raisons qui expliquent qu'une politique interne telle que la politique JAI ait pu devenir un objectif de politique étrangère. Les logiques sécuritaires ne semblent en effet pas suffisantes pour expliquer le développement de cet agenda qui a débuté bien avant le 11 septembre. Par ailleurs, il faudra également davantage d'études qui analysent le contenu et la forme que prend ce nouveau mode de gouvernance avec les voisins de l'UE. Ainsi parviendrons-nous peut-être à surmonter les frontières académiques. 